\section{Review Article}

$\mathrm{J}$ Exp Clin Med

2021; 38(S1): 17-22

doi: $10.52142 /$ omujecm.38.si.gastro. 2

\title{
Treatment of variceal bleeding
}

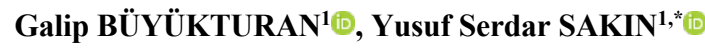

${ }^{1}$ Department of Gastroenterology, Gülhane School of Medicine, University of Health Sciences, Ankara, Turkey

\begin{tabular}{l}
\hline Received: $23.09 .2020 \quad$ Accepted/Published Online: 27.12 .2020 \\
\hline Abstract \\
Variceal bleeding is a gastrointestinal emergency that is one of the major causes of mortality in patients with cirrhosis. A rise in portal pressure \\
(portal hypertension) occurs when there is resistance to outflow from the portal vein. Varices develop to decompress the hypertensive portal vein \\
and return blood to the systemic circulation. A major cause of cirrhosis-related morbidity and mortality is the development of variceal hemorrhage, \\
a direct consequence of portal hypertension. Prevention of bleeding of these variceal veins and treatment after bleeding is an important target. In \\
this article, we will discuss variceal bleeding secondary to portal hypertension and its treatment based on current data.
\end{tabular}

Keywords: variceal bleeding, endoscopic band ligation, endoscopic sclerotherapy

\section{Introduction}

Cirrhosis is termed as the late stage of progressive hepatic fibrosis characterized by the disruption of the hepatic parenchymal structure and the formation of regenerative nodules. Many factors play a role in the etiology of cirrhosis (D'Amico et al., 2006). Currently, mortality is still high and causes significant labor loss. The prognosis of patients with cirrhosis is largely due to its complications. Treatment of cirrhosis is limited except liver transplantation. An important cause of the morbidity and mortality associated with cirrhosis is the development of variceal bleeding secondary to portal hypertension. The prognosis of patients with variceal bleeding depends on the bleeding or other complications are associated with underlying chronic liver disease and its management. The mortality rate due to active variceal bleeding is around 20 percent during each bleeding and re-bleeding is observed in 70 percent of patients within one year. Upper gastrointestinal bleeding unrelated to portal hypertension is also common in patients with portal hypertension (e.g., peptic ulcer disease).

\section{Formation of varices}

Portal vein pressure is determined by the product of the portal flow volume and the resistance to flow out of the portal vein. Portal hypertension (defined as hydrostatic pressure $>5 \mathrm{mmHg}$ ) occurs from the obstruction of proximal portal venous outflow. Occlusion may occur in the presinusoidal (e.g., portal vein thrombosis, portal fibrosis), sinusoidal (e.g., cirrhosis) or postsinusoidal (e.g., veno-occlusive disease, Budd-Chiari syndrome) area. Cirrhosis in the form of obstruction in the sinusoidal region is the most common cause of portal hypertension. In these patients, portal vein pressure increased due to increased resistance to permeability in portal sinusoids and increased portal blood inflow due to splanchnic arteriolar vasodilation. An increase in portal pressure occurs when there is resistance in the outlet tract of the portal vein (portal hypertension). Variceal veins direct blood into the systemic circulation via portocaval shunts to open the hypertensive portal vein and drain the blood. These shunts are the gastroesophageal, paraumbilical, retroperitoneal, intestinal and hemorrhoidal regions. When the pressure gradient between the portal and hepatic veins rises above $12 \mathrm{mmHg}$, variceal veins begin to form. At low pressures, variceal veins do not occur, and they do not bleed (D'Amico et al., 2006; Chang et al., 2020; Egbe et al., 2020). Measuring the difference between portal-hepatic venous pressure gradient, wedge hepatic venous pressure (approximates sinusoidal and portal pressures in cirrhosis) and free hepatic venous pressure can be done by the hepatic venous catheterization method. Measuring by hepatic venous catheterization method does not estimate the size of varices but may be useful for monitoring the success of treatment aimed at lowering portal pressures.

\subsection{Risk of variceal bleeding}

The annual risk of variceal bleeding in patients with varices due to cirrhosis has been found to be five percent and 28 
percent for three years (Merli et al., 2003). Many clinical and physiological factors are used to predict the risk of variceal bleeding in patients with cirrhosis. These are:

- Location of varices

- Size of varices

- Appearance of varices

- Clinical characteristics of the patients

- Variceal pressure

\subsection{Location of varices}

Varices can theoretically be seen in the entire gastrointestinal system from mouth to rectum. But it is often found in portosystemic shunt sites in the esophagus, stomach, and rectum. Varices develop deep within the submucosa in the middle of the esophagus, but gradually become more superficial in the distal esophagus. Therefore, esophageal varices at the gastroesophageal junction have the thinnest supportive tissue layer and there is a high probability of rupture and bleeding (Sarin et al., 2014).

Gastric varices are usually classified according to their location associated with bleeding risks:

Gastroesophageal varices (GOV) type 1; These are variceal veins that directly continue from the small curvature of the stomach with the esophagus.

Gastroesophageal varices (GOV) type 2; These are variceal veins that directly continue with the esophagus from the great curvature of the stomach.

Isolated gastric varices (IGV) type 1; It is less common than GOVs. It is in the fundus of the stomach.

Isolated gastric varices (IGV) type 2; It is found in other locations in the stomach.

Gastroesophageal varices bleeding more frequently than isolated gastric varices, but when IGVs bleed, they bleed more massively. The bleeding incidence of IGV type 1 is high (78 percent). The most common bleeding type is GOV type 2 (Sarin et al., 1992).

\subsection{Size of varices}

The risk of variceal bleeding is related to the diameter (size) of the varices. The explanation for the relationship between variceal size and bleeding risk is derived from Laplace's law; Small increases in vessel radius cause large increases in wall tension. This causes the vessel to burst and bleed. The classification of esophageal varices' size is commonly as follows (Beppu et al.,1981);

- F1: Small, flat varices

- F2: Enlarged, tortuous varices occupying less than a third of the lumen

- F3: Large coil-shaped varices covering more than one third of the lumen
In general, F2 and F3 type varices should be treated. In addition, patients with decompensated cirrhosis should be treated even if their varices' size is small (Garcia-Tsao et al., 2017).

\subsection{Appearance of varices}

Various morphological features of varices observed on endoscopy are associated with an increased risk of bleeding (Kim et al., 1997).

- Cherry red spots

- Red velvet vein appearance

- Hematocystic spots are separate red spots on varices that resemble "blood bubbles"

- Diffuse erythema indicates the diffuse red color of varices

\section{Clinical features of the patients}

CHILD scoring is an indicator of liver dysfunction based on the presence of serum albumin concentration, bilirubin level, prothrombin time, acid, and encephalopathy. A higher score is associated with an increased likelihood of variceal bleeding. A previous history of variceal bleeding is an indicator of an increased risk of bleeding again. For example, only one third of all patients with cirrhosis experience variceal bleeding, while more than 70 percent experience recurrent variceal bleeding after the first bleeding. Bleeding recurs within six weeks in one third of patients with the first bleeding referred to as "early re-bleeding", and after six weeks in one third referred to as "late re-bleeding" (Tripathi et al., 2015). Among the risk factors for early recurrent bleeding are being over 60 years old, kidney failure and ascites (de Francis et al., 1992). The risk of early re-bleeding is greatest immediately after cessation of active bleeding (50 percent of such events occur within 48 hours) and decreases over time.

\subsection{Variceal pressure}

Although measurement of variceal pressure is not routinely performed, it can be accurately measured with a pressure sensitive endoscopic gauge. Variceal pressure may be an important determinant for variceal bleeding. The incidence of variceal bleeding with variceal pressure at different levels is as follows (Bosch et al.,1986);

- $\leq 13 \mathrm{mmHg}-0 / 25$ (0 percent)

->13 and $\leq 14 \mathrm{mmHg}-1 / 11$ (9 percent)

- $>14$ and $\leq 15 \mathrm{mmHg}-2 / 12$ (17 percent)

- $>15$ and $\leq 16 \mathrm{mmHg}-7 / 14$ (50 percent)

-> $16 \mathrm{mmHg}-18 / 25$ (72 percent)

\subsection{Risk classification}

The calculation of an index that numerically measures the risk of variceal bleeding numerically in each patient with CHILD classification, variceal size and the presence of specific appearance can be used to evaluate the prognosis (Table 1) (North Italian Endoscopic Club for the Study and Treatment of 
Esophageal Varices, 1988). The calculated risk is the highest in the first year after these risk factors are identified. As an example, a patient with CHILD class $\mathrm{C}$ cirrhosis and tense ascites and large variceal veins with red marks has an approximately 76 percent chance of developing variceal bleeding within one year. Such a patient is an open candidate for prophylactic treatment to prevent bleeding.

Table 1. Shows the percentage of bleeding within a year according to the characteristics of variceal veins

\begin{tabular}{|l|c|c|c|c|c|c|c|c|c|}
\hline \multicolumn{1}{|c|}{ Percent of variceal bleeding in a year according to its characteristics } \\
\hline $\begin{array}{l}\text { Appearance of } \\
\text { varices }\end{array}$ & \multicolumn{3}{|c|}{$\begin{array}{c}\text { CHILD } \\
\text { CLASS A }\end{array}$} & \multicolumn{3}{c|}{ CHILD } \\
CLASS B & \multicolumn{3}{c|}{$\begin{array}{c}\text { CHILD } \\
\text { CLASS C }\end{array}$} \\
\hline & F1 & F2 & F3 & F1 & F2 & F3 & F1 & F2 & F3 \\
\hline+ & 6 & 10 & 15 & 10 & 16 & 26 & 20 & 30 & 42 \\
\hline+ & 8 & 12 & 19 & 15 & 23 & 33 & 28 & 38 & 54 \\
\hline++ & 12 & 16 & 24 & 20 & 30 & 42 & 26 & 48 & 64 \\
\hline+++ & 16 & 23 & 34 & 28 & 40 & 52 & 44 & 60 & 76 \\
\hline
\end{tabular}

End Stage Liver Disease (MELD) score was also found to predict mortality after acute variceal bleeding. MELD score above 19 is associated with a 20 percent mortality rate, and a MELD score below 19 is associated with a mortality rate of less than five percent. MELD scores have also been shown to predict gastric variceal bleeding (Soga et al., 2009).

\section{Treatment of variceal bleeding}

The prognosis of patients with variceal bleeding depends on achieving hemostasis and avoiding complications associated with underlying chronic liver disease. Treatment of the underlying cause of liver disease reduces the severity of portal hypertension and variceal bleeding. This is most common in patients who quit alcohol and who have a sustained virological response after hepatitis $\mathrm{C}$ treatment. Treatment of acute variceal bleeding usually requires multidisciplinary care including gastroenterology, intensive care, and interventional radiology.

\subsection{Goals of treatment}

- Ensuring and maintaining hemodynamic stability

- Ensuring and maintaining adequate oxygenation

- Control of bleeding

- Prevention of complications

\subsection{Basic treatment}

General resuscitation and supportive measures are applied for patients with a history of variceal bleeding or gastrointestinal bleeding who may have varices (e.g., patients with cirrhosis). To ensure hemodynamic stability, appropriate vascular access is established, and fluid replacement is initiated. Oxygenation is provided with a nasal cannula. Endotracheal intubation may be considered in patients with hemodynamic instability as it will facilitate the endoscopic procedure. Whether endotracheal intubation is effective in preventing aspiration pneumonia is controversial. Typically, nasogastric tube placement followed by gastric lavage can be used to remove particulate matter, fresh blood, and clots from the stomach prior to endoscopy. Typically, a prokinetic agent (e.g., erythromycin) may be given prior to upper endoscopy to improve endoscopic imaging (Pateron et al., 2011). If hemoglobin is $<7 \mathrm{~g} / \mathrm{dL}$, blood transfusion should be performed to keep hemoglobin between $\geq 7 \mathrm{~g} / \mathrm{dL}$ and $<9 \mathrm{~g} / \mathrm{dL}$. However, it should be aimed to keep hemoglobin at $9 \mathrm{~g} / \mathrm{dL}$ for patients at high risk of mortality and morbidity, such as patients with comorbid disease such as unstable coronary artery disease or with ongoing active bleeding. Prophylactic antibiotic therapy is recommended in patients with cirrhosis presenting with gastrointestinal bleeding because antibiotic prophylaxis reduces the risk of death, infection, and bleeding (Chavez-Tapia et al., 2011). Typically, parenteral ceftriaxone therapy is administered $(1 \mathrm{~g}$ intravenous daily for seven days). The patient is discharged with oral ciprofloxacin treatment. For hospitalized patients, prophylactic antibiotics reduce the risk of mortality, infection (e.g., spontaneous bacterial peritonitis, urinary tract infections) and bleeding.

\section{Treatment}

Current treatment options for acute variceal bleeding include medications (vasopressin, somatostatin, and analogs), endoscopy, transjugulary intrahepatic portosystemic shunt (TIPS) and surgical treatment.

\subsection{Medical therapy}

The first step to stop variceal bleeding is pharmacological treatment. Vasoactive drugs reduce portal blood flow and are used for the treatment of acute variceal bleeding. It includes vasopressin, somatostatin, and its analogs (terlipressin and octreotide). As a group, it has been shown that vasoactive drugs reduce mortality and develop hemostasis in patients with acute variceal bleeding (Wells et al., 2012). Pharmacological treatment should be initiated considering variceal bleeding in all patients with upper gastrointestinal bleeding in patients with varices or suspected portal hypertension (e.g., patients with cirrhosis). Pharmacological treatment should continue until it is determined that the source of bleeding is not from varices. Terlipressin should be considered first in pharmacological treatment. It is administered at an initial dose of $2 \mathrm{mg}$ IV every four hours and can be titrated up to $1 \mathrm{mg} I V$ every four hours after bleeding has been controlled. Pharmacological treatment typically continues for three to five days after the bleeding has ceased. Terlipressin is the most effective drug for mortality (Ioannou et al., 2003). When used in addition to sclerotherapy, treatment with somatostatin or octreotide infusions is superior to sclerotherapy alone or somatostatin alone for prevention of premature re-bleeding and possibly survival (D'Amico et al.,1998; Besson et al., 1995; Avgerinos et al., 1997). Combination therapy with somatostatin analogues and endoscopic variceal ligation has a higher five-day success rate compared to endoscopic variceal ligation alone. Ocreotide is only useful when combined with endoscopic therapy. However, no effect on mortality was shown (D'Amico et al., 2002). 


\subsection{Endoscopic therapy}

It is the definitive treatment method for active variceal bleeding. Two types of endoscopic treatments are commonly used: endoscopic variceal ligation (EVL) and endoscopic sclerotherapy (ES). EVL is generally preferred as the first treatment. If the first treatment attempt with EVL fails, ES can be tried. The aim should be to perform an upper GIS endoscopy after fluid resuscitation and within 12 hours after admission to the hospital.

- EVL is like hemorrhoidal taping; It involves placing small elastic bands around the variceal veins.

- ES involves the injection of sclerosing solution into variceal veins. There are a number of sclerosing solutions available, all of which are effective. The volume and frequency of injections varies widely among endoscopists. 1-2 $\mathrm{mL}$ of 5 percent sodium morrhuate can be used per injection for a total of 12 to $20 \mathrm{~mL}$ per session. Other commonly used alternatives are polidocanol and cyanoacrylate excisions (Lux et al., 1997).

\subsection{TIPS (Transjugulary intrahepatic portosystemic shunt) and surgery}

If the bleeding cannot be controlled endoscopically, a second endoscopic procedure can be performed. Subsequently, treatment options include TIPS placement or surgical shunting. Hemostasis is achieved following TIPS placement in more than ninety percent of patients. In addition, early TIPS placement following endoscopic treatment may increase non-transplant survival in patients with advanced cirrhosis (Lv et al., 2019a; Conejo et al., 2018; Lv et al., 2019b). Absolute contraindications for TIPS placement include heart failure, polycystic liver disease, severe pulmonary hypertension, uncontrolled systemic infection, sepsis, and severe tricuspid regurgitation. Relative contraindications include hepatocellular carcinoma (especially if it is central), portal vein thrombosis, and severe coagulopathy or thrombocytopenia. Complications of TIPS placement include portosystemic encephalopathy, technical complications (e.g., cardiac arrhythmias, liver capsule injury) and TIPS stenosis.

\subsection{Balloon tampon}

It is an option to temporarily stop bleeding from esophageal or stomach varices, but is associated with serious complications, including esophageal rupture. Balloon tamponade appears to be less successful in patients who fail pharmacological therapy and patients with premature bleeding. Esophageal stent placement (Danis stent) has begun to be used as an alternative to balloon tamponade in patients with uncontrolled acute variceal bleeding, and the stent has been replaced by tamponade due to its lower risk of complications and the possibility of feeding while the stent can remain for a long time.

\subsection{Surgical treatment}

It is extremely effective at stopping bleeding and preventing re-bleeding but is associated with a mortality rate of up to 50 percent. It is generally not used because TIPS has a high success rate and lower complication rates. Despite hemostasis, many patients die of postoperative liver failure and surgical complications. Those with severe bleeding, tense ascites, coma, aspiration pneumonia, kidney failure or sepsis are particularly at risk of surgery. The ideal patient for surgical treatment is a person with well-preserved liver function who cannot pass the emerging endoscopic treatment and who does not have bleeding or complications from endoscopy (Rikkers et al., 1995).

Treatment for bleeding gastric varices is cyanoacrylate injection, if possible. However, it is not approved for the treatment of bleeding in gastric varices. If cyanoacrylate injection is not an option, TIPS is done. Varices can sometimes develop in areas other than the stomach and esophagus, and when they bleed, they become clinically important. These are called ectopic varices. Examples are duodenal, rectal, and peristomal varices (developing around the stoma in patients with colostomy). TIPS or surgery is the most appropriate treatment for patients with ectopic variceal bleeding. Endoscopic treatment is usually unsuccessful in ectopic varices.

\subsection{Complications of variceal bleeding}

Hemostasis is achieved with treatment in most patients with variceal bleeding; however, patients with cirrhosis are at risk of complications during hospitalization. Major complications include pneumonia, sepsis, chronic/acute liver failure, hepatic encephalopathy, and kidney failure. All complications are more common when the endoscopic procedure is performed as an emergency rather than elective.

\subsection{Prophylaxis of variceal bleeding}

In the presence of variceal veins, prophylaxis is applied to prevent bleeding. Prophylaxis of variceal bleeding is divided into two as primary and secondary prophylaxis.

\subsection{Primary prophylaxis}

Primary prophylaxis aims to prevent variceal bleeding in patients with esophageal varices without a history of bleeding. Pre-Primer prophylaxis refers to the measures to prevent the development of varices. In patients with such portal hypertension but without variceal veins, treatment of the underlying liver disease may help prevent the development of variceal veins. However, treatment with beta blockers is not recommended in patients without variceal veins (Groszmann et al., 2005). Measures to prevent bleeding in patients with a history of variceal bleeding are called secondary prophylaxis. We aim to achieve one of two results in primary prophylaxis;

- Reduction of portal hypertension (e.g., beta blockers, surgical portal decompression or transjugular intrahepatic shunts)

- Direct treatment of variceal veins (e.g., variceal ligation)

Endoscopic screening for esophageal varices should be performed in most of the patients with cirrhosis, so prophylactic treatment can be given in case of high risk of 
bleeding varices. Scanning is typically done with an upper GIS endoscopy. Screening is repeated every two to three years in patients with compensated cirrhosis without varices. Screening is repeated every two years in patients with small variceal veins. Endoscopy is repeated every year in patients with decompensated cirrhosis or in the presence of endoscopic evidence of increased bleeding risk in variceal veins. At these periodic controls, patients with CHILD B or C disease, medium-large size variceal veins, and patients with red color changes in varices should receive primary prophylaxis. Patients with CHILD B or C disease or with red discoloration of varices can be treated with beta blockers, but if they cannot tolerate EVL can be applied. EVL is preferred primarily in patients with medium-large variceal veins ( $\mathrm{Li}$ et al., 2011).

In general, for patients treated with non-selective betablockers, nadolol can be used starting with a dose of $40 \mathrm{mg}$ per day (as it can be given once a day). Propranolol may also be preferred in this regard. The hemodynamic goal of treatment with a non-selective beta blocker is to reduce the hepatic venous pressure gradient (HVPG) to 10 percent or $\leq 12 \mathrm{mmHg}$. If HVPG cannot be used to monitor therapy, the beta blocker dose can be titrated by targeting a heart rate of about 55 to 60 beats per minute. Patients with decompensated cirrhosis (Child $\mathrm{B}$ or $\mathrm{C}$ ) receiving beta blockers should be closely monitored for side effects. Beta blockers may need to be stopped if the patient becomes resistant to treatment, develops hepatic encephalopathy or spontaneous bacterial peritonitis. Approaches not recommended for primary prophylaxis include nitrates (alone or in combination with beta-blockers), shunt therapy, or sclerotherapy. In addition, combination therapy with beta blockers and EVL is not recommended for primary prophylaxis (de Franchis et al., 2010).

\subsection{Secondary prophylaxis}

Measures to prevent bleeding in patients with a history of variceal bleeding are called secondary prophylaxis (Shi et al., 2013). Patients recovering from the first episode of esophageal variceal bleeding experience a high rate of bleeding (up to 60 percent during the first year).

- Acute variceal bleeding: The acute bleeding episode consists of the time interval from admission to the hospital to the $120^{\text {th }}$ hour $\left(5^{\text {th }}\right.$ day).

- Variceal re-bleeding: Variceal bleeding describes the bleeding occurring $\geq 120$ hours after the first bleeding, provided that initial hemostasis can be achieved.

Liver transplantation is recommended for patients with a MELD score of 14 and a history of esophageal variceal bleeding because transplantation is the most effective longterm treatment for variceal bleeding and other complications of portal hypertension (Zhang et al., 2009). Patients are evaluated for interventions that prevent the progression of liver disease. So, treatment of the underlying disease is important. For patients with cirrhosis recovering from an attack of esophageal variceal bleeding, EVL is preferred to prevent re-bleeding rather than endoscopic sclerotherapy. EVL is performed one to two weeks after discharge from the hospital and then every two to four weeks until variceal veins disappear. A nonselective beta blocker is started on the $5^{\text {th }}$ day after the first bleeding period. The duration of beta blocker therapy varies depending on the patient's course of disease, the development of side effects and the clinician's preference.

In patients who are contraindicated or intolerant to beta blockers, only EVL is recommended to prevent recurrent variceal bleeding. TIPS placement is the preferred long-term strategy for patients with cirrhosis who initially recover from variceal bleeding but later develop recurrent variceal bleeding despite EVL and / or beta blocker therapy (Propst et al., 1995; Westaby et al., 1992).

\section{Conclusion}

Among patients with cirrhosis, variceal veins occur at a rate of 5 to 15 percent per year, and variceal bleeding develops in one third of patients with varices. Variceal bleeding is a gastrointestinal emergency that is one of the main causes of death in patients with cirrhosis. The prognosis of patients with variceal bleeding depends on achieving hemostasis and avoiding complications associated with the underlying chronic liver disease. Treatment options available for acute variceal bleeding include medications (vasopressin, somatostatin, and analogs), endoscopy, TIPS and surgery. An important issue as well as intervention in variceal bleeding is the primary and secondary measures that will prevent bleeding after variceal formation and bleeding again after bleeding.

\section{Conflict of interest}

All authors declare no conflict of interest.

\section{References}

1. Avgerinos, A., Nevens, F., Raptis, S., Fevery, J., 1997. Early administration of somatostatin and efficacy of sclerotherapy in acute oesophageal variceal bleeds: The European Acute Bleeding Oesophageal Variceal Episodes (ABOVE) randomised trial. Lancet. 350,1495-1499.

2. Beppu, K., Inokuchi, K., Koyanagi, N., Nakayama, S., Sakata, H., Kitano, S., Kobayashi, M., 1981. Prediction of variceal hemorrhage by esophageal endoscopy. Gastrointest. Endosc. 27, 213-218.

3. Besson, I., Ingrand, P., Person, B., Boutroux, D., Heresbach, D., Bernard, P., Hochain, P., Larricq, J., Gourlaouen, A., Ribard, D., et al., 1995. Sclerotherapy with or without octreotide for acute variceal bleeding. N. Engl. J. Med. 333, 555-560.

4. Bosch, J., Bordas, JM., Rigau, J., Viola, C., Mastai, R., Kravetz, D., Navasa, M., Rodés, J., 1986. Noninvasive measurement of the pressure of esophageal varices using an endoscopic gauge: comparison with measurements by variceal puncture in patients undergoing endoscopic sclerotherapy. Hepatology. 6, 667-672.

5. Chang, Y., Suk, K.T., Jeong, S.W., Yoo, J.J., Kim, S.G., Kim, Y.S., Lee, S.H., Kim, H.S., Kang, S.H., Baik, S.K., Kim, D.J., Kim, M.Y., Jang, J.Y., 2020. Application of hepatic venous pressure gradient to predict prognosis in cirrhotic patients with a low model for end-stage liver disease score. Diagnostics. 10, 805.

6. Chavez-Tapia. NC., Barrientos-Gutierrez, T., Tellez-Avila, F., Soares-Weiser, K., Mendez-Sanchez, N., Gluud, C., Uribe, M., 2011. Meta-analysis: antibiotic prophylaxis for cirrhotic patients 
with upper gastrointestinal bleeding - an updated Cochrane review. Aliment. Pharmacol. Ther. 34, 509-518.

7. Conejo, I., Guardascione, M.A., Tandon, P., Tandon, P., Cachero, A., Castellote, J., Abraldes, J.G., Amitrano, L., Genescà, J., Augustin, S., 2018. Multicenter external validation of risk stratification criteria for patients with variceal bleeding. Clin. Gastroenterol. Hepatol. 16, 132-139.

8. D’Amico, G., Politi, F., Morabito, A., D'Antoni, A., Guerrera, D., Giannuoli, G., Traina, M., Vizzini, G., Pasta, L., Pagliaro, L., 1998. Octreotide compared with placebo in a treatment strategy for early rebleeding in cirrhosis. A double blind, randomized pragmatic trial. Hepatology. 28, 1206-1214.

9. D’amico, G., Criscuoli, V., Fili, D., Mocciaro, F., Pagliaro, L., 2002. Meta-analysis of trials for variceal bleeding. Hepatology. 36, 1023-1024.

10. D’Amico, G., Garcia-Pagan, J.C., Luca, A., Bosch, J., 2006. Hepatic vein pressure gradient reduction and prevention of variceal bleeding in cirrhosis: A systematic review. Gastroenterology. 131,1611-1624.

11. de Franchis, R., Primignani, M., 1992. Why do varices bleed? Gastroenterol. Clin. North Am. 21, 85-101.

12. de Franchis, R., Faculty, B.V., 2010. Revising consensus in portal hypertension: Report of the Baveno $\mathrm{V}$ consensus workshop on methodology of diagnosis and therapy in portal hypertension. J. Hepatol. 53, 762-768.

13. Garcia-Tsao G, Abraldes JG, Berzigotti A, Bosch J., 2017. Portal hypertensive bleeding in cirrhosis: Risk stratification, diagnosis, and management: 2016 practice guidance by the American Association for the study of liver diseases. Hepatology. 65, 310335 .

14. Ioannou, G., Doust, J., Rockey, D.C., 2003. Terlipressin for acute esophageal variceal hemorrhage. Cochrane Database Syst. Rev. CD002147.

15. Egbe, A.C., Miranda, W.R., Veldtman, G.R., Graham, R.P., Kamath PS., 2020. Hepatic venous pressure gradient in fontan physiology has limited diagnostic and prognostic significance. CJC Open. 5, 360-364.

16. Groszmann, R.J., Garcia-Tsao, G., Bosch, J., Grace, N.D., Burroughs, A.K., Planas, R., Escorsell, A., Garcia-Pagan, J.C., Patch, D., Matloff, D.S., Gao, H., Makuch, R., Portal Hypertension Collaborative Group., 2005. Beta-blockers to prevent gastroesophageal varices in patients with cirrhosis. N. Engl. J. Med. 353, 2254-61.

17. Kim, T., Shijo, H., Kokawa, H., Tokumitsu, H., Kubara, K., Ota, K., Akiyoshi, N., Iida, T., Yokoyama, M., Okumura, M., 1997. Risk factors for hemorrhage from gastric fundal varices. Hepatology. 25, 307-312.

18. Li, L., Yu, C., Li, Y., 2011 Endoscopic band ligation versus pharmacological therapy for variceal bleeding in cirrhosis: A meta-analysis. Can. J. Gastroenterol. 25, 147-155.

19. Lux, G., Retterspitz, M., Stabenow-Lohbauer, U., et al. 1997. Treatment of bleeding esophageal varices with cyanoacrylate and polidocanol, or polidocanol alone: results of a prospective study in an unselected group of patients with cirrhosis of the liver. Endoscopy. 29(4), 241-246.

20. Lv, Y., Yang, Z., Liu, L., AVB-TIPS Study Group., 2019a Early TIPS with covered stents versus standard treatment for acute variceal bleeding in patients with advanced cirrhosis: a randomised controlled trial. Lancet. Gastroenterol. Hepatol. 4,
587-598.

21. Merli, M., Nicolini, G., Angeloni, S., Rinaldi, V., De Santis, A., Merkel, C., Attili, AF., Riggio, O., 2003. Incidence and natural history of small esophageal varices in cirrhotic patients. J. Hepatol. 38, 266-272.

22. North Italian Endoscopic Club for the Study and Treatment of Esophageal Varices., 1988. Prediction of the first variceal hemorrhage in patients with cirrhosis of the liver and esophageal varices. A prospective multicenter study. N. Engl. J. Med. 319, 983-989.

23. Pateron, D., Vicaut, E., Debuc, E., Sahraoui, K., Carbonell, N., Bobbia, X., Thabut, D., Adnet, F., Nahon, P., Amathieu, R., Aout, M., Javaud, N., Ray, P., Trinchet, JC., HDUPE Collaborative Study Group., 2011. Erythromycin infusion or gastric lavage for upper gastrointestinal bleeding: A multicenter randomized controlled trial. Ann. Emerg. Med. 57, 582-589.

24. Propst, A., Propst, T., Zangerl, G., Ofner, D., Judmaier, G., Vogel, W., 1995. Prognosis and life expectancy in chronic liver disease. Dig. Dis. Sci. 40, 1805-1815.

25. Rikkers, L.F., Jin, G., 1995. Emergency shunt. Role in the present management of variceal bleeding. Arch. Surg. 130, 472-479.

26. Sarin, S.K., Lahoti, D., Saxena, S.P, Murthy, N.S., Makwana, U.K., 1992. Prevalence, classification, and natural history of gastric varices: A long-term follow-up study in 568 portal hypertension patients. Hepatology. 16, 1343-1349.

27. Sarin, S.K., Kumar, A., 2014. Endoscopic treatment of gastric varices. Clin. Liver. Dis. 18, 809-827.

28. Singal, A.G., Volk, M.L., Jensen, D., Di Bisceglie, A.M., Schoenfeld, P.S., 2010. A sustained viral response is associated with reduced liver-related morbidity and mortality in patients with hepatitis C virus. Clin. Gastroenterol. Hepatol. 8, 280-288.

29. Shi, K.Q., Liu, W.Y., Pan, Z.Z., Ling, X.F., Chen, S.L., Chen, Y.P., Fan, Y.C., Zheng, M.H., 2013. Secondary prophylaxis of variceal bleeding for cirrhotic patients: A multiple-treatments meta-analysis. Eur. J. Clin. Invest. 43, 844-854.

30. Soga, K., Tomikashi, K., Miyawaki, K., Fukumoto, K., Wakabayashi, N., Konishi, H., Mitsufuji, S., Yoshida, N., Kokura, S., Naito, Y., Kataoka, K., Yoshikawa, T., 2009. MELD score, child-pugh score, and decreased albumin as risk factors for gastric variceal bleeding. Hepatogastroenterology. 56, 1552-1556.

31. Tripathi, D., Stanley, AJ., Hayes, PC., Patch, D., Millson, C., Mehrzad, H., Austin, A., Ferguson, JW., Olliff, SP., Hudson, M., Christie, JM., Clinical Services and Standards Committee of the British Society of Gastroenterology., 2015. U.K. guidelines on the management of variceal haemorrhage in cirrhotic patients. Gut. $64,1680-1704$

32. Wells, M., Chande, N., Adams, P., Beaton, M., Levstik, M., Boyce, E., Mrkobrada, M., 2012. Meta-analysis: Vasoactive medications for the management of acute variceal bleeds. Aliment. Pharmacol. Ther. 35,1267.

33. Westaby, D., 1992. Prevention of recurrent variceal bleeding: endoscopic techniques. Gastrointest. Endosc. Clin. North. Am. 2,120-125.

34. Zhang, J.Y., Kuai, J.H., Jia, J.D., Wang, B.E., Qin, C.Y., 2009. The effect of portal hypertension on prognosis in patients with decompensated liver cirrhosis. Zhonghua. Gan. Zang. Bing. Za. Zhi. 263-265. 